\title{
Blastocystis hominis microbiota: study of I 3255 patients and review of the literature
}

\begin{abstract}
Summary
Blastocystis $s p$ is the most common intestinal parasite found in humans. Its role in human health is widely discussed due to the high proportion of asymptomatic carriers. Hence the interest of our study to objectify the number of subjects infested by this parasite in our population. This is a retrospective study carried out over a period of ten years, from January 2009 until December 2018, in the parasitology-mycology laboratory of the Avicenna military hospital in Marrakech. 13,255 subjects were included with at least one parasitological examination of the stool, of which 2,799 examinations were positive, with a simple parasite index of $21.11 \%$. Blastocystis hominis was found in 675 subjects with a specific parasite index of $5.09 \%$, and it was co-associated in 446 subjects with a specific parasite index (IPSp) of 3.36\%. The most frequent association was that of Blastocystis hominis and E. histolytica/dispar with a rate of $(25.5 \%)$ of all polyparasitic patients followed by the combination of Blastocystis hominis and Entamoeba coli $(20.8 \%)$, the combination of Blastocystis hominis and Endolimax nana with a rate of $(16,1 \%)$ and lastly Blastocystis hominis and Entamoeba hartmani (5.3\% In patients with polyparasitism, the association of Blastocystis hominis with Entamoeba coli was $20.8 \%$, with Endolimax nana with a rate of $16.1 \%$ and finally with Entamoeba hartmani with a prevalence of $5.3 \%$. It is always necessary to insist on strict observance of the rules of prophylaxis against oral-fecal contamination
\end{abstract}

Volume 9 Issue 2 - 202।

\author{
J Belkhair,' I Karrati,' M Tarmidi,' M El \\ Mezouari,' R Moutaj² \\ 'Service of Parasitology Mycology, Avicenna Military Hospital, \\ Morocco \\ ${ }^{2} \mathrm{Head}$ of the Parasitology and Mycology Department,Avicenna \\ Military Hospital, Morocco
}

Correspondence:] Belkhair, Service of Parasitology Mycology, Avicenna Military Hospital, Marrakech, Morocco, Tel +2 12694289 104, Email belkhairjihane0 I@gmail.com

Received: February 12, 202 I | Published: March 12, 202 |

\section{Introduction}

Blastocystis spp is a eukaryotic protozoan. It is a single-celled intestinal parasite found in the intestinal tract of humans and various animals. It is clear that most carriers of Blastocystis hominis appear asymptomatic, its place in human health remains very controversial: commensal or opportunistic. ${ }^{1}$ Our work will try to answer this question by studying the experience of the mycology parasitology department of the Avicenna military hospital in Marrakech on this parasite.

\section{Materials and methods}

This is a retrospective analytical descriptive study, based, on the files of all patients presenting to the Parasitology and Mycology department of the Avicenne Military Hospital in Marrakech for a parasitological examination. The study was conducted over a period of 10 years extending from January 2009 until December 2018, including all patients presenting to the Parasitology-Mycology department of the HMA of Marrakech for a parasitological examination of the stool. The sample is systematically taken while respecting several "EPS reference" conditions. Three parasitological examinations of the stool at intervals of 2-3 days were examined. Each lot is subjected to a macroscopic, direct microscopic and microscopic examination after concentration. Ritchie technique and MIF technique-Bailanger (ready-to-use kit).

\section{Statistical analysis}

The data were entered using Microsoft Office Word 2016 software. Statistical analysis was performed using Microsoft Office Excel 2016 software. The various parameters were calculated and analyzed. Qualitative variables are expressed as a percentage, while quantitative variables are expressed as an average.

\section{Results}

Our sample contained 13,255 patients. The average age was 28.5 years old. The sex ratio $\mathrm{M} / \mathrm{F}$ of our adult sample was 2.33 . During the period of the study between January 2009 and December 2018, the population examined annually increased between 922 cases examined in 2009 and 1034 examined in 2018, with a peak in the years 20132014 (Figure 1 'thesis') Distribution of the population examined by year).

The simple parasite index was $21.11 \%(2799 / 13255)$. The parasite index for the years of the study was varying between 15.41 in 2011 being the lowest index and 28.41 in 2010 being the highest index (Table 1). During this study period, the number of parasitized patients was 2799 patients. This included 1928 males $(68.88 \%)$ and 871 females $(31.11 \%)$, with a sex ratio $(\mathrm{M} / \mathrm{F})$ of 2.21 . The P.I. of female patients $(21.97 \%)$ was higher than that of males $(20.77 \%)$.

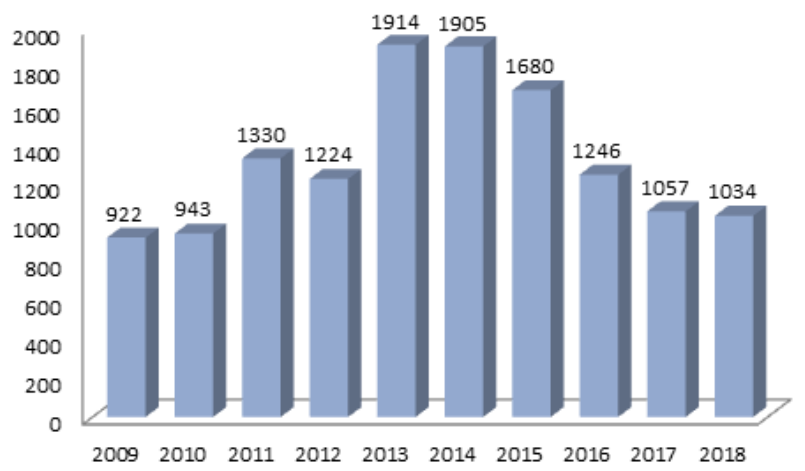

Figure I Distribution of the population examined by year.

During this study, 675 subjects confirmed the presence of $B$. hominis (in its two cystic and vegetative forms) out of 2799 positive analyzes with a specific parasite index (IPSp) (the percentage of subjects parasitized by a specific parasite compared to the total number of subjects examined) by $5.09 \%$. The $B$. hominis infestation was clearly on the increase over the ten years of this study. During the ten years of this study, the frequency of positive carriage of $B$. hominis was sharply increasing with a peak around the year 2015 . 
Table I Simple parasite index according to year

\begin{tabular}{|c|c|c|c|c|c|c|c|c|c|c|c|}
\hline & 2009 & 2010 & 2011 & 2012 & 2013 & 2014 & 2015 & 2016 & 2017 & 2018 & Total \\
\hline Number of patients examined & 922 & 943 & 1330 & 1224 & 1914 & 1905 & 1680 & 1246 & 1057 & 1034 & 13255 \\
\hline Number of parasitized subjects & 169 & 268 & 205 & 204 & 289 & 405 & 431 & 351 & 228 & 249 & 2799 \\
\hline Annual parasite index (\%) & 18.32 & $28.4 I$ & $15.4 \mid$ & 16.66 & 15.09 & 21.25 & 25.65 & 28.17 & 21.57 & 24.08 & 21.11 \\
\hline
\end{tabular}

During this study, B. hominis has been co-associated with a parasite in up to four parasites, in 446 subjects During this study, it was found in 446 subjects; the $B$. hominis micro-organism has been co-associated with one parasite or even four parasites, out of a number

Table 2 Different co-association of Blastocystis hominis with other parasites

\begin{tabular}{|c|c|}
\hline Different associations & Parasitic subjects \\
\hline B. hominis + E. histolyticaldispar & 114 \\
\hline B. hominis + E. coli & 93 \\
\hline B. hominis + E. nana & 72 \\
\hline B. hominis + E. coli + E. histolyticaldispar & 35 \\
\hline B. hominis + E. hartmani & 24 \\
\hline B. hominis+ E. histolytica histolytica & 18 \\
\hline B. hominis + E. nana+ E. histolytica/dispar & 17 \\
\hline B. hominis + E.H.H + E. histolyticaldispar & 15 \\
\hline B. hominis + E. coli+ E.H.H+ E. H. dispar & 6 \\
\hline B. hominis + E. coli+ E. nana+ E. H. dispar & 6 \\
\hline B. hominis $+E$. coli $+E$. hartmani & 5 \\
\hline B. hominis +Giardia intestinalis & 5 \\
\hline B. hominis + E. coli+ E.nana & 4 \\
\hline B. hominis $+\mathrm{CH}$. Mesnili & 4 \\
\hline B.hominis + E.coli + E.histolytica histolytica & 3 \\
\hline B. hominis $+P$. butschlii & 2 \\
\hline B. hominis + E. hartmani+T. Intestinalis & 2 \\
\hline B. hominis + E. histolyticaminuta & 2 \\
\hline B. hominis+ E. nana+ E.H.H+ E. H. dispar & 2 \\
\hline B. hominis + G.I. + E. histolyticadispar & 2 \\
\hline B. hominis + E. nana+ E. hartmani & 2 \\
\hline B. hominis + Levures & 2 \\
\hline B. hominis + E. coli $++\mathrm{CH}$. mesnili & 1 \\
\hline B. hominis + E. coli+ E. nana+ E. hartmani & $\mathrm{I}$ \\
\hline B. hominis $+E$. coli $+P$. butschlii & I \\
\hline B. hominis+ E. hartmani+ E. H. dispar & 1 \\
\hline B. hominis + E. hartmani + E.H.H & 1 \\
\hline B. hominis+ E. hartmani+ G. Intestinalis & $\mathrm{I}$ \\
\hline B. hominis + E.H.H + Euf d'A. lumbricoïdes & $\mathrm{I}$ \\
\hline B. hominis+ E.H.H. + E. H. dispar & 1 \\
\hline B. hominis + E. nana+ E. hartmani & 1 \\
\hline B.H+G. I. + E. coli+ E. nana + E. hartmani & I \\
\hline B. hominis $+G$. Intestinalis $+P$. butschlii & 1 \\
\hline
\end{tabular}

of 2799 subjects parasitized with a specific parasite index (IPSp) of $3.36 \%$. The different associations were 33 associations in 446 subjects composed of 143 Women and 303 Men (Table 2).

The B. hominis-E. histolytica/dispar association was found in 114 subjects, with a male predominance of 83 male subjects against 31 female subjects during the ten years of the study from 2014 to 'in the year 2018 with a peak in the year 2015 (Figure 2). The $B$. hominis $-E$. coli association was found 93 times, in 62 men and 31 women during the ten years of our study (Figure 3). The B. hominis-E. nana association was found in 72 subjects, with a male predominance of 52 male subjects against 20 female subjects during the ten years of the study from 2012 until the year 2018 with a peak in the year 2015 (Figure 4).

The association of $B$. hominis $-E$. coli- E. histolytica/dispar was found in 35 subjects over the ten years in the years 2014,2015 / 2016/2017 and 2018 with a female predominance. The B.hominisChilomastixmesnili combination was found 4 times during the ten years of our study in the years 2014, 2015 and 2018. The B. hominis-E. Histolytica Dispar-C. mesnili combination was found twice in a man and a woman during the ten years of our study in the years 2014 and 2018.

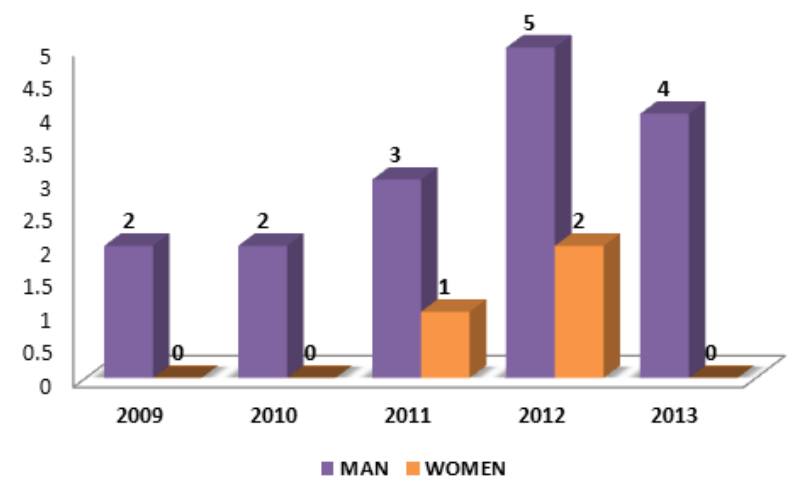

Figure 2 Annual frequency by sex of the Blastocystishominis-Entamoeba Histolytica/Dispar co-association.

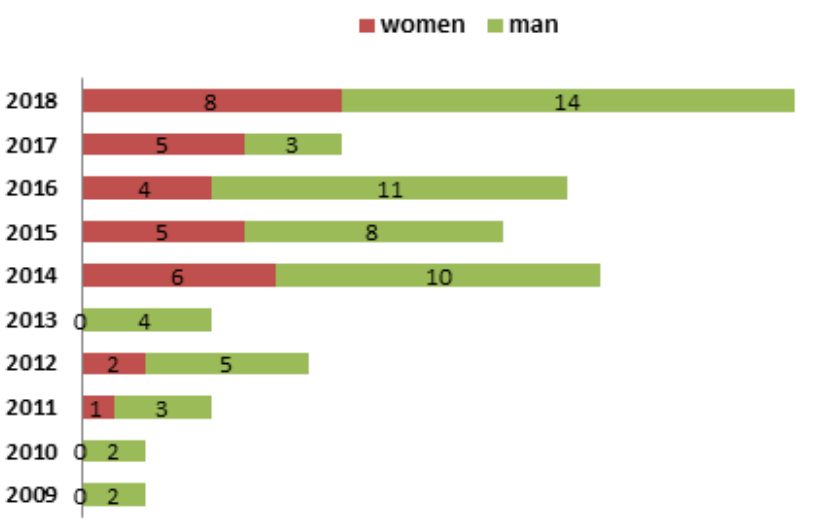

Figure 3 Annual frequency by sex of the B. hominis-E. coli co-association. 


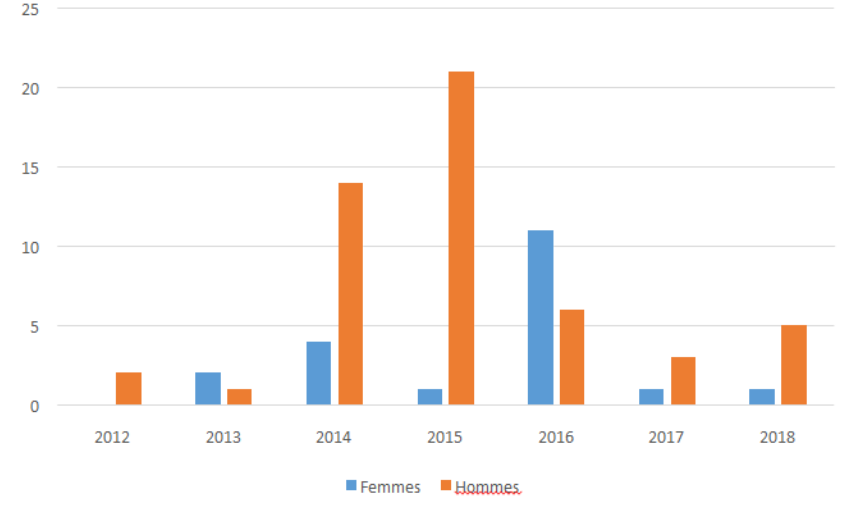

Figure 4 Annual frequency by sex of the co-association of B. h- E. nana.

\section{Discussion}

Blastocystis $s p$ is the most common intestinal parasite found in humans. Although first described over 100 years ago, there remain many gray areas about its biology and epidemiology. Its role in human health is widely discussed due to the high proportion of asymptomatic carriers. However, recent studies have shown that its prevalence is vastly underestimated in the general population, especially in patients with irritable bowel syndrome. Several studies have been carried out throughout the world in order to know the epidemiological profile of $B$. hominis infestations in populations.

The sex ratio $(\mathrm{M} / \mathrm{F})$ of our adult sample was 2.33 , which means the significant male representation in our study unlike the study in Tunisia (sex ratio of 0.94), or that in France (sex ratio of 1.37)., ${ }^{2,3}$ The very low female representativeness is due to the fact that the majority of military structures employed men. In fact, several studies have not found a significant difference in parasite infection rates between the two sexes. ${ }^{4}$ In our series the mean age of cases was 28.5. Data on educational level, place of residence and economic status were not collected; our results agree with the data reported by Salvador ${ }^{5}$ and by Losada ${ }^{6}$; the average age of 36 , and 31 respectively.

The overall parasite prevalence rate found in our study was $21.11 \%$. It is very close to the rate recorded in A.'s study. Benouis ${ }^{7}$ with a rate of $19.96 \%$. Or again in the Masmoudi series ${ }^{8}$ carried out in Sfax, Tunisia where the rate was $21.4 \%{ }^{9}$ objected to a prevalence of $18.1 \%$, using PCR as a means of diagnosis Benouis et al., ${ }^{7}$ found a rate of $19.96 \%$, Masmoudi et al., ${ }^{8}$ shown a rate of $21.4 \%{ }^{9}$ however, Trabelsi et al., ${ }^{2}$ shown a prevalence of $18.1 \%$, using PCR as a means of diagnosis. The Specific Parasite Index (SPI) in our study was $5.09 \%$. Our results are in agreement with the results of the series of Trabelsi ${ }^{2}$ which authenticates a prevalence of $7.27 \%$, and also coincides with the results of the series of Bouree ${ }^{1}$ which showed an index $6.08 \%$ specific for Blastocystis spp. In addition, the results of the Chabaa ${ }^{9}$ series demonstrated a strong infestation with Blastocystis $s p$. compared to the results of our series with a specific IPS of $13.39 \%$. Losada, ${ }^{6}$ found a higher rate of infection with Blastocystis spp with a specific prevalence of $18 \%$. On the other hand, Beyhan, ${ }^{10}$ claimed the infestation with Blastocystis spp over $0.55 \%$. As a result, this low rate recorded in our series could be explained by an improvement in the rules of hygiene within our population. In the contrast, quality control of sanitation and piping has improved the conditions. The water; and finally geo-climatic conditions which are not conducive to the development of this protozoan in our region has had a check on the clinical manifestation. The $B$. hominis parasite has a much higher prevalence in countries with a tropical climate $(25.7 \%$ in Malaysia and $32.6 \%$ in China). ${ }^{11,12}$
Among the polyparasites patients in our study, $71.3 \%$ had a combination of two parasites and $21 \%$ had a combination of three intestinal parasites, and $7.6 \%$ had a combination of four intestinal parasites. The most frequent association was that of $B$. hominis and E. histolytica/dispar with a rate of $25.5 \%$ of all polyparasitic patients followed by the combination of B. hominis and E. coli (20.8\%), the combination of $B$. hominis and $E$. nana with a rate of $16.1 \%$ and lastly the association B. hominis and E. hartmani (5.3\%). It should be noted that the presence of parasitic association shows a low level of sanitary, food and fecal hygiene as well as the unfavorable living conditions for these polyparasitic subjects. ${ }^{13}$ The triple associations were: as $B$. hominis $+E$. coli $+E$. histolytica/dispar $(7.8 \%)$, followed by $B$. hominis $+E$. nana $+E$. histolytica dispar (3.8\%). And B. hominis $+E$. histolytica histolytica $+E$. histolytica dispar (3.3\%). The study of the correlation between sex and the type of B. hominis-parasite association did not show much interest since it was not significant. The B. hominis infestation associated with other parasites can be said to be unrelated to sex. This data is confirmed by other studies., ${ }^{71}$ Chabaa $^{9}$ demonstrated parasitism, the most frequent association of which was with non-pathogenic amoeba in $50 \%$ of cases. In the series by Trabelsi, ${ }^{2} B$. hominis was associated with a parasite in $40.6 \%$. The main associated parasites were Endolimax nana (40.4\%), E. coli (20.2\%), Giardia intestinalis (8.7\%), E. hartmanni (6.7\%). Salvador et al., ${ }^{5}$ objectified polyparasitism at $61.9 \%$, the main associated parasites were Entamoeba fragilis (16.2\%), E. nana (15.8\%), and E. $\operatorname{coli}(12.4 \%)$

The part of the responsibility of $B$. hominis in the genesis of the clinical symptoms is difficult to apprehend insofar as it is very often associated with numerous other parasites and intestinal infectious agents. Is this a pathogen or is it just opportunistic? Some authors have established a correlation between an abundant presence of $B$. hominis found in the stool (more than 5 blastocysts per microscopic field) and the appearance of digestive disorders. When no other etiology is found, it may be assumed that $B$. hominis is responsible for the symptoms. In addition, there are strains of $B$. hominis of different pathogenicity depending on their subtype. Subtype 1 is always associated with symptomatic people unlike subtype 2 which is always associated with asymptomatic people. ${ }^{15}$ Subtype 3 , which is the most common subtype, and subtype 4 are found in both symptomatic and asymptomatic people, in equal proportions with regard to subtype 3. According to these recent Observations, the pathogenicity of $B$. hominis would depend not on the abundance of the parasite in the stool but rather on the virulence of the subtype. Subtype 2 constitutes a nonpathogenic $B$. hominis genotype while subtype 1 is pathogenic. As for subtype 3 , it would have a potential pathogenic effect. However, all the etiologies of diarrhea are not systematically investigated. A significant proportion of patients with $B$. hominis also harbor other enteric parasites, viral or bacterial agents. Some known pathogens are not always looked for. It is therefore important to carry out a complete parasitological examination, unfortunately not carried out in practice.

Repeating stool examinations (for example three successive samples three days apart) makes it possible to find parasites ( $E$. histolytica, G. intestinalis, E. nanus) or other pathogens that may explain digestive disorders. ${ }^{1}$ There is also a possibility of finding other unidentified organisms responsible for digestive symptoms. This parasite causes digestive disorders in people with weakened immune systems without any other etiology being found; in this case it would therefore have an opportunist character. Other authors do not establish a relationship between $B$. hominis and symptoms. This parasite is found in healthy people and is generally considered to be a commensal since its presence in the stools of asymptomatic 
subjects can reach a frequency of $16 \%{ }^{16}$ Pathogenicity criteria were thus determined: ${ }^{1}$-the finding of a large number of $B$. hominis, greater than 5 by microscopic field (at $100 \mathrm{X}$ magnification) - the presence of clinical signs- and especially the absence of other known etiology of diarrhea and digestive disorders (viral, bacterial, parasitic, etc.). The meeting of these three criteria leads to the diagnosis of blastocystosis. Therefore, treatment should be initiated to try to correct intestinal dysmicrobism and limit the development of $B$. hominis. The question of opportunism, however, seems clearer. High prevalence's of the parasite in patients with HIV have been found, with clinical pictures of blastocystosis in the most immunosuppressed patients. In these populations, several studies have shown a correlation between the prevalence of Blastocystis sp. and a CD4 T lymphocyte count of less than $200 / \mathrm{mm} 3 .{ }^{17}$ Cases of blastocystosis have also been reported in kidney transplant recipients as well as in patients with malignant hemopathy. ${ }^{18,19}$ We also find in the literature studies with contradictory conclusions regarding the relationship between the parasitic subtype and symptomatology. There is currently no evidence indicating that one subtype is more virulent than another, which suggests instead the existence of variable virulence within the same subtype and, potentially, host factors.

In our study, studying the pathogenicity of B. hominis was difficult in the absence of clinical data from the patients examined and the retrospective nature of our study. The mycological parasitology laboratory of the Avicenna Military Hospital performs parasitological examinations of the stool as part of a clinical diagnosis, aptitude visit and systematic control of the cooks.

\section{Conclusion}

Blastocystis spp has long been regarded as a simple commensal. Recent epidemiological and experimental data seem to demonstrate that we must remain cautious about this precept. The long-term effect of Blastocystis on the intestinal epithelia as well as on the gut microbiota should be explored. The high prevalence of this parasitosis with faecal-oral transmission in different populations imposes the need for new well-constructed studies to deepen our knowledge on the understanding of the role of Blastocystis in human health.

\section{Acknowledgments}

None.

\section{Conflicts of interest}

Authors declare that there is no conflict of interest.

\section{References}

1. Bouree P. Blastocystis: commensal or pathogenic? Study of 590 cases and review of the literature. Antibiotiques. 2007;9(1):20-24.

2. Trabelsi S, BenHaj Ali I, Khaled S. Epidemiological and clinical characteristics of Blastocystis hominis. La Tunisie Medicale. 2010;88(3):190-192.
3. El Safadi D, Cian A, Nourrisson C, et al. Prevalence, risk factors for infection and subtype distribution of the intestinal parasite Blastocystis sp. from a large-scale multi-center study in France. BMC Infect Dis. 2016;16(1):451.

4. Incani R, Ferrer E, Hoek D, et al. Diagnosis of intestinal parasites in a rural community of Venezuela: advantages and disadvantages of using microscopy or RT-PCR. Acta Trop. 2017;167:64-70.

5. Salvador F, Sulleiro E, Sánchez-Montalvá A, et al. Epidemiological and clinical profile of adult patients with Blastocystis sp. infection in Barcelona, Spain. Parasit Vectors. 2016;9(1):548.

6. Ocana-Losada C, Cuenca-Gómez JA, Cabezas-Fernández MT, et al. Clinical and epidemiological characteristics of intestinal parasite infection by Blastocystishominis. Rev Clin Esp. 2018;218(3):115-120.

7. Benouis A, Bekkouche Z, Benmansour Z. Epidemiological study of human intestinal parasitosis in the Hospital of Oran (Algeria). International Journal of Innovation and Applied Studies. 2013;2(4):613-620.

8. Masmoudi M, Hajjaji D, Sallami H, et al. Dépistage de parasitisme intestinal chez les manipulateurs de denrées alimentaires : à propos de 417 cas. Archives des Maladies Professionnelles et de l'Environnement. 2012;73(3):425-442.

9. Chabaa L, Tliguit H, Khalloufi AS, et al. Blastocystis hominis: étude de la prévalence dans les populations Marocaines. Maroc Médical. 2000;22(3):186-189.

10. Beyhan YE, Yilmaz H, Cengiz ZT, et al. Clinical significance and prevalence of Blastocystis hominis in Van, Turkey. Saudi Med J. 2015;36(9):1118-1121.

11. Abdulsalam AM, Ithoi I, Al-Mekhlafi HM, et al. Drinking water is a significant predictor of Blastocystis infection among rural Malaysian primary school children. Parasitology. 2012;139(8):1014-1020.

12. Li LH, Zhou XN, Du ZW, et al. Molecular epidemiology of human Blastocystis in a village in Yunnan province, China. Parasitol Int. 2007;56(4):281-286.

13. Control of tropical diseases. World Health Organization; 1998.

14. Fischer P, Sohail M. Blastocystis hominis and travelers. Travel Med Infect Dis. 2005;3(1):33-38.

15. Clark C, Stensvold C, Suresh G, et al. Terminology for Blastocystis subtypes-a consensus. Trends Parasitol. 2007;23(3):93-96.

16. Gaye C. Etude d"un protozoaire parasite: Blastocystis hominis. Thèse de doctorat: Pharmacie. Toulouse: Université Paul Sabatier; 1995. 102 p.

17. Cirioni O, Giacometti A, Drenaggi D, et al. Prevalence and clinical relevance of Blastocystis hominis in diverse patient cohorts. Eur $J$ Epidemiol. 1999;15(4):389-393.

18. Ghosh K, Ayyaril M, Nirmala V. Acute GVHD involving the gastrointestinal tract and infestation with Blastocystis hominis in a patient with chronic myeloid leukaemia following allogeneic bone marrow transplantation. Bone Marrow Transplant. 1998;22(11):1115-1117.

19. Rao K, Sekar U, Iraivan KT, et al. Blastocystis hominis-an emerging cause of diarrhoea in renal transplant recipients. J Assoc Physicians India. 2003;51:719-721 